\title{
Bilateral Cranial IX and X Nerve Palsies After Mild Traumatic Brain Injury
}

\author{
Seung Don Yoo, MD, PhD, Dong Hwan Kim, MD, PhD, Seung Ah Lee, MD, PhD, \\ Hye In Joo, MD, Jin Ah Yeo, MD, Sung Joon Chung, MD
}

Department of Rehabilitation Medicine, Kyung Hee University, Seoul, Korea

We report a 57-year-old man with bilateral cranial nerve IX and X palsies who presented with severe dysphagia. After a mild head injury, the patient complained of difficult swallowing. Physical examination revealed normal tongue motion and no uvular deviation. Cervical X-ray findings were negative, but a brain computed tomography revealed a skull fracture involving bilateral jugular foramen. Laryngoscopy indicated bilateral vocal cord palsy. In a videofluoroscopic swallowing study, food residue remained in the vallecula and pyriform sinus, and there was reduced motion of the pharynx and larynx. Electromyography confirmed bilateral superior and recurrent laryngeal neuropathy.

Keywords Basilar skull fracture, Cranial nerve diseases

\section{INTRODUCTION}

A skull base fracture can cause damage to lower cranial nerves as they traverse the skull. However, the incidence of traumatic lower cranial nerve palsies is relatively rare [1]. A variety of syndromes involving lower cranial nerves is described $[1,2]$. These are commonly unilateral, and only a few cases of bilateral cranial nerve involvement are reported. We report a case of skull base fracture involving both the jugular foramen, which was clinically associated with cranial nerve IX and X palsies with severe dysphagia

Received April 20, 2015; Accepted June 3, 2015

Corresponding author: Sung Joon Chung

Department of Rehabilitation Medicine, Kyung Hee University, 892 Dongnam-ro, Gangdong-gu, Seoul 05278, Korea

Tel: +82-2-440-7246, Fax: +82-2-440-7260, E-mail: sungjoon.chung@ gmail.com

(c) This is an open-access article distributed under the terms of the Creative Commons Attribution Non-Commercial License (http://creativecommons. org/licenses/by-nc/4.0) which permits unrestricted noncommercial use, distribution, and reproduction in any medium, provided the original work is properly cited.

Copyright $\odot 2016$ by Korean Academy of Rehabilitation Medicine as the chief complaint.

\section{CASE REPORT}

A 57-year-old male was sent to our emergency department after falling down in a drunken state. He had no particular history of underlying diseases. On arrival, he was nearly alert and well oriented. His initial Glasgow Coma Scale score was 14/15. He complained of a headache in the back of the head and had bleeding in his nose and oral cavity. On initial assessment, there was no weakness and sensory loss. The strength of the sternocleidomastoid and deltoid was intact and no deviation of tongue was detected. Anteroposterior, lateral and open mouth view of the cervical radiograph showed no abnormal findings. Therefore, a brain computed tomography scan was performed and revealed a subarachnoid hemorrhage, pneumocephalus in the prepontine, and a fracture of the base of skull extending bilaterally to the jugular foramina (Fig. 1). He was transferred to the neu- 

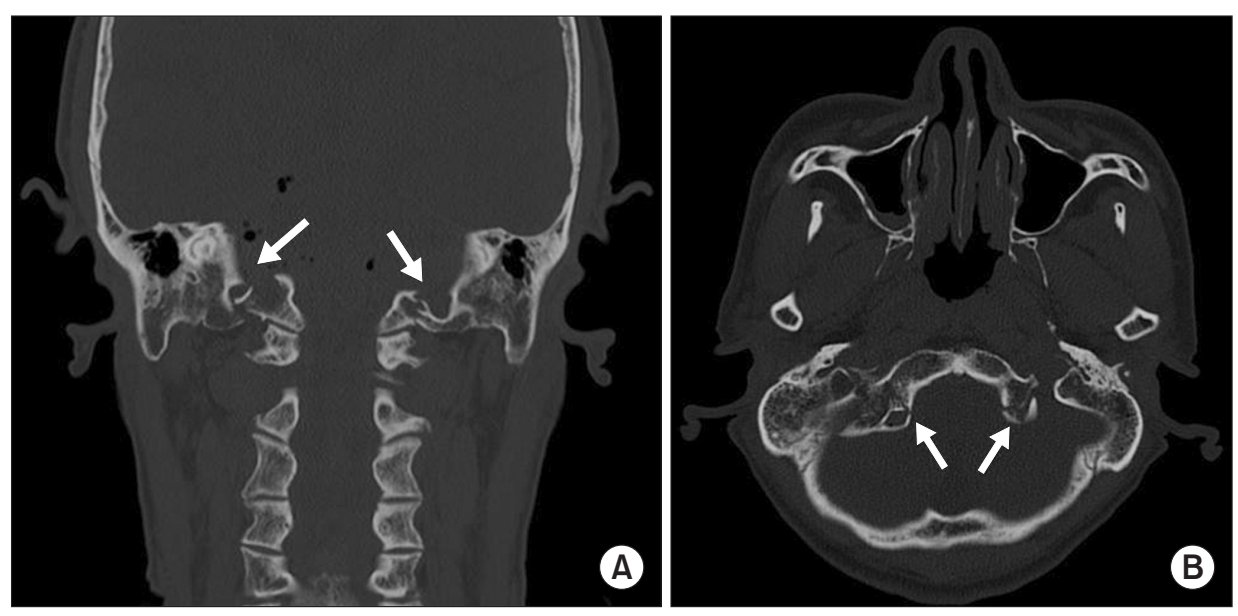

Fig. 1. Transverse (A) and coronal (B) computed tomography revealed bilateral occipital condylar fracture (arrow) extending to the jugular foramina.
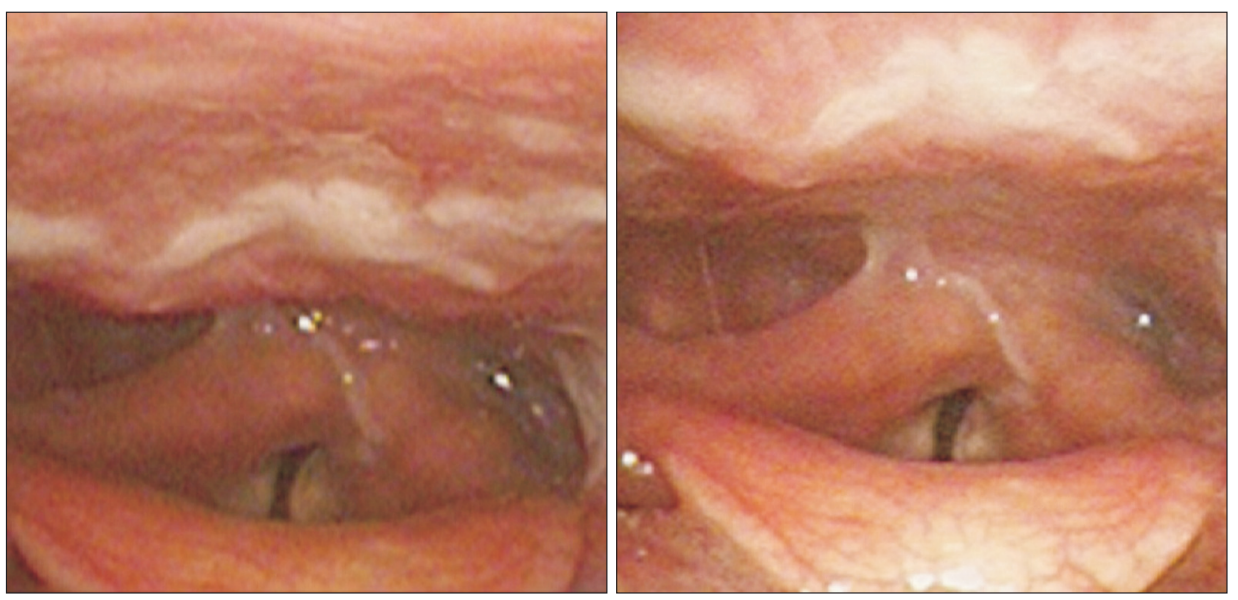

Fig. 2. Laryngoscopy showed incomplete movement of bilateral vocal cord.

rosurgery department for close observation. On day 1 , he complained of pharyngeal discomfort and difficulty in swallowing. Additional symptoms included dyspnea and voice change. On laryngoscopy, we could confirm bilateral vocal cord palsy with saliva pooling in both pyriform sinuses (Fig. 2). A nasogastric tube was applied for enteral feeding because of the high risk of aspiration pneumonia.

On day 5, he was transferred to the rehabilitation department for further management. He was stable and did not show any additional neurologic deficits except for dysphagia. The sensation of posterior tongue was decreased and the gag reflex was absent. In comparison, the tongue movement was relatively normal. Videofluoroscopic swallowing study (VFSS) results indicated decreased laryngeal and pharyngeal motility. A large amount of residue was retained in the vallecular pouch (Fig. 3), and swallowing was not intact. After 4 weeks, laryngeal electromyography was performed jointly by a physiatrist and well-trained resident. Both sides of cricothyroid muscle and thyroarytenoid were tested. The study revealed positive sharp waves in the right thyroarytenoid muscle. The interference pattern showed discrete at both cricothyroid and thyroarytenoid muscles. Bilateral cranial IX and X nerves palsies was confirmed based on the collective findings confirm.

The patient underwent occupational therapy for dysphagia. Pharyngeal stimulation was conducted and exercise for increasing pharyngeal muscle contraction force was applied. The patient was educated on vocal cord adduction exercise. Four-week follow-up VFSS and laryngoscopy revealed mild improvement of left vocal cord motility and laryngeal elevation, but pharyngeal muscle weakness was constant. Percutaneous endoscopic gastrostomy was performed at 8 weeks. VFSS at 1 year showed better results than previous tests but aspiration with large amount of residue persisted. 

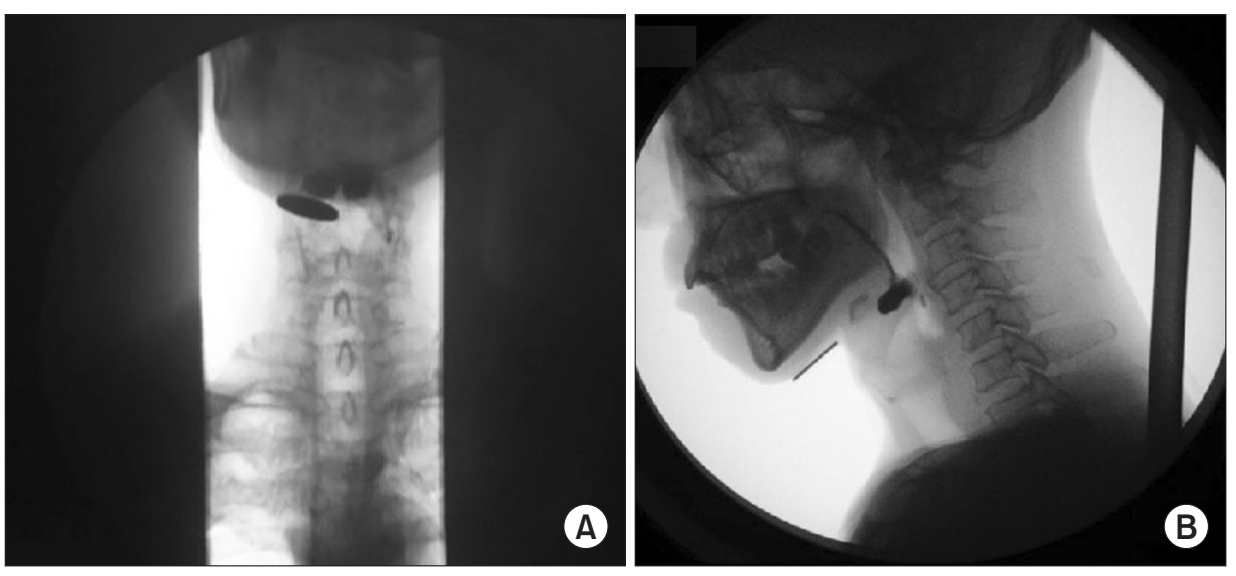

Fig. 3. The videofluoroscopic swallowing study showed a large amount of vallecular residue due to absence of the swallowing reflex: (A) anteroposterior view and (B) lateral view.

\section{DISCUSSION}

Traumatic lower cranial nerve neuropathy first described by Collet et al. involved cranial IX, X, XI and XII injury that caused pharyngeal scapular glossal laryngeal hemiplegia after gunshot injury [3]. Subsequently, there have been several reports of various traumatic lower cranial neuropathy that usually followed a fracture through the jugular foramen or occipital condyle fracture (OCF) [4]. Cranial IX, X and XI nerves exit the cranial vault through the jugular foramen and cranial nerve XII exits from the hypoglossal canal, next to the occipital condyle [5]. Thus, the anatomical location of jugular foramen and OCF can account for the frequent occurrence of lower cranial nerve deficits following these fractures. These cranial nerve injuries can be caused by even mild traumatic injury with similar distribution to severe head injury [6]. The clinical symptoms can present variedly according to the nerve involvements, and may include immediate or delayed dysphonia, swallowing difficulty, or respiratory distress. The mechanisms of injury are direct nerve compression by displaced bone fragments and ischemia related nerve edema could account for delayed palsies [4]. Lesions in this area result in characteristic syndromes. The Collet-Sicard syndrome is one of the syndromes of multiple lower cranial palsies characterized by dysfunction of cranial IX, X, XI and XII nerves [3].

Although multiple lower cranial nerve palsies related to a skull base fracture are relatively well known, only a few cases with isolated cranial IX and X nerve palsies are reported [7,8]. Urculo et al. [8] reported the first case of isolated cranial IX and X nerves palsy in a patient with OCF who had unilateral IX and X nerves paralysis with delayed presentation. The isolated injury of cranial nerve IX and X nerve was demonstrated in other reports of variable anatomy of cranial nerve XI and a variety of interconnections leading to variable symptoms of lesions at the same anatomic location [9]. Bozkurt et al. [10] proposed that the nerve course and number of rootlets are possible mechanisms of isolated IX and X nerve palsies. The cranial IX and $\mathrm{X}$ nerves emerging from brain stem are more perpendicular, as compared to the cranial XI nerve; and the number of rootlets of the cranial XI nerve is higher than those of the cranial IX and X nerves.

Bilateral cranial IX and X nerve palsies are much rarer than unilateral palsies. The only case reported in literature is by Lehn et al. [2] who described a patient with OCF complicated by bilateral palsies of cranial IX and X nerve. The patient had dramatic clinical manifestations including postural hypotension, dysphagia, severe upper gastrointestinal dysmotility and airway obstruction, which posed a severe management challenge to the physicians.

Compared to previous reports of bilateral cranial IX and $\mathrm{X}$ palsies, our patient presented relatively mild clinical manifestation except for dysphagia. Altered oral secretion or severe postural hypotension could not be detected in our case. On the other hand, our patient showed high blood pressure for 2 months despite no previous history of hypertension. It is likely that the severity of injury of involved nerves could lead to different clinical presentations. Both cases of bilateral palsies showed immediate onset as opposed to unilateral cranial XI and X nerve palsies, which usually present in delayed form. The neurologic deficits of the 2 cases of bilateral palsies persisted over the course of 1 year. 
In conclusion, we report a rare case of traumatic bilateral cranial XI and X palsies with severe dysphagia. Since patients with mild traumatic brain injury could be affected, hidden skull fracture should be considered in patients with lower cranial neuropathy.

\section{CONFLICT OF INTEREST}

No potential conflict of interest relevant to this article was reported.

\section{REFERENCES}

1. Legros B, Fournier P, Chiaroni P, Ritz O, Fusciardi J. Basal fracture of the skull and lower (IX, X, XI, XII) cranial nerves palsy: four case reports including two fractures of the occipital condyle: a literature review. J Trauma 2000;48:342-8.

2. Lehn AC, Lettieri J, Grimley R. A case of bilateral lower cranial nerve palsies after base of skull trauma with complex management issues: case report and review of the literature. Neurologist 2012;18:152-4.

3. Sharma BS, Mahajan RK, Bhatia S, Khosla VK. ColletSicard syndrome after closed head injury. Clin Neurol Neurosurg 1994;96:197-8.
4. Tuli S, Tator CH, Fehlings MG, Mackay M. Occipital condyle fractures. Neurosurgery 1997;41:368-77.

5. Wen HT, Rhoton AL Jr, Katsuta T, de Oliveira E. Microsurgical anatomy of the transcondylar, supracondylar, and paracondylar extensions of the far-lateral approach. J Neurosurg 1997;87:555-85.

6. Coello AF, Canals AG, Gonzalez JM, Martin JJ. Cranial nerve injury after minor head trauma. J Neurosurg 2010;113:547-55.

7. Capuano C, Costagliola C, Shamsaldin M, Maleci A, Di Lorenzo N. Occipital condyle fractures: a hidden nosologic entity. An experience with 10 cases. Acta Neurochir (Wien) 2004;146:779-84.

8. Urculo E, Arrazola M, Arrazola M Jr, Riu I, Moyua A. Delayed glossopharyngeal and vagus nerve paralysis following occipital condyle fracture: case report. J Neurosurg 1996;84:522-5.

9. Brown H. Anatomy of the spinal accessory nerve plexus: relevance to head and neck cancer and atherosclerosis. Exp Biol Med (Maywood) 2002;227:5708.

10. Bozkurt G, Hazer B, Yaman ME, Akbay A, Akalan N. Isolated paralysis of glossopharyngeal and vagus nerve associated with type II occipital condyle fracture: case report. Childs Nerv Syst 2010;26:719-22. 